\title{
OPCIONES REALES E INCENTIVOS
}

\author{
Esteban Colla De Robertis*
}

(Recibido: Octubre 2011 / Aprobado: Enero 2012)

\section{Resumen}

Administrar eficientemente una opción real implica establecer si conviene ejecutar el proyecto de beneficios inciertos, o diferir la ejecución al futuro, cuando aparece nueva información útil y la incertidumbre sobre los beneficios se reduce o desaparece. Con frecuencia esta decisión se basa en la recomendación de un experto, con capacidad para determinar la rentabilidad del proyecto en caso de ejecutarse. En el presente trabajo analizamos el contrato óptimo que el dueño de la opción real debe proponer al experto para que involucre recursos costosos que le permitan recabar información de utilidad para decidir sobre el timing óptimo, y para que efectúe una recomendación consistente con la información recabada.

Palabras clave: opciones reales, información asimétrica, incentivos, contratos, agencia, experto

Clasificación JEL: D82, D86, D92, L26

\begin{abstract}
Efficient management of a real option involves establishing the convenience of undertaking a proyect with uncertain benefits versus deferring decision

* Universidad Panamericana, Escuela de Ciencias Económicas y Empresariales. Correo electrónico: <ecolla@up.edu.mx>.

Se agredecen los comentarios y sugerencias de Andrei Gomberg y César Martinelli. Cualquier error del presente trabajo es exclusiva responsabilidad del autor.
\end{abstract}


and waiting for new useful information to appear and uncertainty to dissapear. Frequently, this decision is based on the recommendation of an expert, with the ability to determine the benefits of the proyect if it is undertaken. In this paper we analyze the optimal contract that the owner of the real option should propose to the expert in order to give her incentives to use costly resources in order to acquire useful information, and to make a recommendation which is consistent with the information acquired.

Keywords: real options, asymmetric information, incentives, contracts, agency, expert

JEL Classification: D82, D86, D92, L26

\section{Introducción}

Buena parte de los proyectos de inversión que se llevan a cabo en una economía están caracterizados por la confluencia de tres factores: (i) La inversión necesaria para ejecutar el proyecto es irreversible (por ejemplo, cuando el capital invertido en el proyecto no puede recuperarse porque los activos tienen alto grado de especificidad). (ii) Es posible postergar la ejecución del proyecto. (iii) Los beneficios del proyecto son inciertos y pueden variar a lo largo del tiempo. Proyectos con estas características suelen recibir el nombre de opciones reales, por la analogía con las opciones financieras. El dueño de una opción real tiene el derecho de ejecutar un proyecto en algún momento a lo largo de un periodo, pero no está obligado a ejecutarlo, del mismo modo que el dueño de una opción financiera tiene el derecho a realizar una determinada operación sobre un activo subyacente, en o antes de una fecha de vencimiento, pero no está obligado a realizar dicha operación.

Ejercer en el presente una opción real involucra un tradeoff: se obtienen beneficios inmediatos pero se pierde la posibilidad de ejecutar el proyecto en el futuro, con el costo de oportunidad asociado si los beneficios son mayores. Cuando los beneficios del proyecto, en caso de ejecutarse, dependen de alguna variable observable (por ejemplo precios de mercado), la regla de decisión suele involucrar un umbral: llevar e cabo el proyecto solo si la variable observada es mayor que un determinado valor umbral, y esperar en caso contrario (McDonald y Siegel, 1986; Dixit y Pindyck, 1994). La espera tiene valor porque puede permitir resolver incertidumbre sobre 
los beneficios futuros del proyecto, pero también tiene costo de oportunidad, que corresponde a los beneficios perdidos por no ejecutar inmediatamente el proyecto. La regla óptima es la que logra un balance adecuado entre los beneficios y los costos de esperar. La industria del petróleo y la industria farmacéutica son dos ejemplos de industrias en las que las carteras de proyectos de las firmas que las conforman tienen un fuerte componente de opciones reales. (Para otros casos referirse a Dixit y Pindyck, 1994; Smit y Trigeorgis, 2004, presentan casos en los que el ejercicio de la opción real depende de la interacción estratégica entre varias firmas).

Las opciones reales en las que nos enfocamos en el presente trabajo son aquellas en las que los beneficios subyacentes (en caso de ejecutarse el proyecto) no son fácilmente observables. Tal es el caso, por ejemplo, del desarrollo de nuevos productos cuya demanda es incierta y difícilmente estimable. En estos casos, para la decisión de ejecutar o esperar suele ser útil contar con la opinión de un experto, que cuenta con una tecnología (know-how) para estimar el beneficio subyacente del proyecto (usualmente involucrando recursos costosos o incurriendo en desutilidades debido al esfuerzo). En tales situaciones, el dueño de la opción real deberá diseñar un contrato que incentive al experto a esforzarse por conocer con la mayor precisión posible los beneficios subyacentes, y que también incentive al experto a comunicar verazmente dicha información.

Gromb y Martimort (2007), desde ahora GM, analizan en detalle los incentivos a expertos, y la posibilidad de colusión entre ellos. GM definen a un experto como un agente que realiza una acción o esfuerzo para obtener determinada información. Tanto la acción como la información adquiridas son privadas del experto y no verificables, por lo que no pueden exigirse en contratos. Como consecuencia, los incentivos dados a los expertos tienen como finalidad inducirlos a que se esfuercen por adquirir información, y a revelarla verazmente; sin embargo, no pueden requerir del experto el reporte del esfuerzo ni de la información adquirida. GM consideran contratos que especifican una recomendación del experto y un pago, que puede (y óptimamente debe) estar condicionado a cualquier variable observable que se produzca al implementar la recomendación del experto, y estudian la forma del contrato que incentiva al experto a esforzarse y a efectuar una recomendación en línea con la información adquirida, a modo de administrar eficientemente el proyecto. El modelo es de un solo periodo y las dos decisiones posibles son ejecutar o abandonar el proyecto. 
A diferencia del modelo de un solo periodo considerado por GM, en este trabajo consideramos una opción real cuya administración eficiente requiere decidir entre ejecutar inmediatamente un proyecto con beneficios inciertos, o esperar a que se resuelva la incertidumbre. El inversor puede delegar la búsqueda de información útil en un experto, con capacidad para obtener una señal informativa pero imperfecta sobre los beneficios del proyecto. El diseño del contrato óptimo entre el inversor y el experto es el objetivo del presente trabajo, para lo cual construimos un modelo sencillo basado en el de GM. Mostramos que si el experto recomienda la ejecución inmediata, el contrato establece una recompensa sólo si el proyecto tiene éxito. Si en cambio el experto recomienda postergar la decisión en espera de mayor información, el pago en el segundo periodo debe hacerse contingente a la información que se revela al postergar el proyecto. Además, mostramos que el pago esperado al experto es mayor que su costo de esfuerzo, ya que es necesaria una prima para incentivar al experto a revelar la información adquirida efectuando una recomendación consistente.

En la siguiente sección presentamos el modelo. En la sección 3 obtenemos el contrato óptimo y ofrecemos un análisis del mismo. Por último presentamos las conclusiones. Todas las demostraciones se relegan al Apéndice.

\section{Modelo}

El modelo considera un inversor con derecho a ejecutar un proyecto irreversible de beneficios inciertos. La inversión puede efectuarse en dos periodos posibles: $t=1,2$. En cada periodo hay dos estados de la naturaleza posibles: el estado favorable $\left(S_{t}=H\right)$ y el estado desfavorable $\left(S_{t}=L\right)$. Concretamente, suponemos que en el estado favorable, el proyecto reporta ganancias $G>0$, y en el estado desfavorable reporta pérdidas iguales a $P$.

El estado de la naturaleza no es observable en el primer periodo, pero es observable en el segundo periodo. Es decir, la incertidumbre en el segundo periodo desaparece. Este supuesto nos permite modelar de forma estilizada el tradeoff esencial de una opción real: ejecutar inmediatamente versus esperar la llegada de más información. En el primer periodo, un experto tiene acceso a una señal correlacionada con el estado de la naturaleza, condicional a la realización de esfuerzo, de costo $e>0$. Este costo puede interpretarse directamente como la desutilidad del experto debido al esfuerzo, o como el costo de los recursos utilizados por la tecnología para recabar información. La precisión de la 
señal si el experto se esfuerza es $\operatorname{Pr}\left(s=S_{1} \mid e\right)=\theta$. Suponemos $1 / 2<\theta<1$; es decir, la señal es informativa pero imperfecta. El estado de la naturaleza en cada periodo está caracterizado por las probabilidades no condicionales $\operatorname{Pr}\left(S_{1}=H\right)=\lambda$ y $\operatorname{Pr}\left(S_{1}=L\right)=1-\lambda$, y por las probabilidades condicionales $\lambda_{H}=\operatorname{Pr}\left(S_{2}=H \mid S_{1}=H\right)$, que es la probabilidad de permanencia en el estado favorable, y $\lambda_{L}=\operatorname{Pr}\left(S_{2}=L \mid S_{1}=L\right)$, que es la probabilidad de permanencia en el estado desfavorable. Las probabilidades no condicionales en el segundo periodo se denotan $\operatorname{Pr}\left(S_{2}=H\right)=\Lambda$ y $\operatorname{Pr}\left(S_{2}=L\right)=1-\Lambda$. La probabilidad no condicional de la señal $s$, se denota $p(s)=\operatorname{Pr}\left(s \mid S_{1}=H\right) \lambda+\operatorname{Pr}\left(s \mid S_{1}=L\right)(1-\lambda)$, la probabilidad de que el estado de la naturaleza en $t$ sea el favorable dada la señal $s$ se denota $q_{t}(s)=\operatorname{Pr}\left(S_{t}=H \mid s\right)$, y la probabilidad de que la señal sea la favorable, condicional al estado de la naturaleza $S_{t^{\prime}}$, se denota $\vartheta_{t}(S)=\operatorname{Pr}\left(s=H \mid S_{t}=S\right)$.

Tanto el inversor como el experto son neutrales al riesgo (maximizan beneficios esperados) y el experto tiene responsabilidad limitada, por lo que el contrato no puede especificar penalidades o pagos del experto al inversor. (Si el experto no tuviera responsabilidad limitada, la administración eficiente puede implementarse a través de un contrato que equivale a vender los derechos de propiedad sobre la opción real al experto.)

El timing es el siguiente:

1. El inversor propone un contrato al experto, y el experto decide si aceptarlo o no aceptarlo.

2. La naturaleza determina el estado $S_{1}$.

3. El experto decide si observa una señal correlacionada con el estado de la naturaleza.

4. El experto efectúa una recomendación al inversor respecto de la ejecución del proyecto. La recomendación puede ser esperar o ejecutar inmediatamente.

5. Si el proyecto se ejecuta inmediatamente, se observan los beneficios, y el inversor paga al experto lo estipulado en el contrato, en función de los beneficios observados.

6. Si la recomendación del experto es esperar, la naturaleza determina el estado $S_{2^{\prime}}$ observado por todos.

7. Si el estado en el segundo periodo es favorable, se ejecuta el proyecto; si es desfavorable, se abandona el proyecto.

8. El inversor paga al experto lo estipulado en el contrato, en función de la decisión del inversor de ejecutar o abandonar. 


\section{El contrato óptimo}

La señal recibida por el experto es información privada de él, y por lo tanto no es verificable. El estado de la naturaleza en el primer periodo no es observable, y en el segundo periodo sí es observable, pero no es verificable. La idea es que un tercero -árbitro o juez- no puede, o le resulta muy costoso, certificar dicha información. En cambio, el resultado del proyecto, si se ejecuta, es información pública y además es verificable por terceros (por ejemplo, mediante la auditoría de los estados contables). En consecuencia, el contrato no puede especificar acciones o pagos condicionales a la señal o al estado de la naturaleza, pero puede especificar pagos contingentes a acciones de cada parte y a información que pueda ser verificable por terceros. En nuestro contexto, el contrato exige al experto una recomendación (ejecutar inmediatamente o diferir para el periodo siguiente), y exige al inversor un pago al experto en el primer periodo si el experto recomienda ejecutar inmediatamente, condicionado a los beneficios del proyecto. Denotamos a estos pagos $t_{1 H}$ y $t_{1 L}$. Además el contrato especifica un pago al experto en el segundo periodo, si su recomendación es diferir, condicionado a la decisión del inversor de ejecutar o abandonar el proyecto. Al desaparecer la incertidumbre, el inversor puede conocer con certeza si el proyecto es rentable $\left(S_{2}=H\right)$ o no $\left(S_{2}=L\right)$, y utilizar esta información para decidir si lo ejecuta o lo abandona (en la jerga financiera "deja morir la opción"). Suponemos que el estado de la naturaleza no es verificable por terceros (por ejemplo por un juez en caso de disputas), aún cuando en el segundo periodo sea información pública. Por lo tanto, los pagos no pueden condicionarse a dicho estado. Sin embargo, el contrato puede especificar pagos en función de acciones verificables del inversor. Como las acciones del inversor en el segundo periodo son ejecutar o dejar morir la opción, y óptimamente estas decisiones se toman respectivamente si el estado de la naturaleza es favorable o desfavorable, el inversor puede en la práctica pagar al experto en función del estado. Denotamos a estos pagos por $t_{2} \mathrm{H}_{\mathrm{y}} t_{2} L$.

A efectos de formular las restricciones de incentivos que debe verificar el contrato, denotamos por $\Pi(R)$ al valor actual de los pagos esperados por el experto si su recomendación es $R$ (= Ejecutar o Diferir) sin haber observado la señal, y denotamos por $\Pi(R \mid S)$ el pago esperado si el experto recomienda ejecutar o diferir habiendo observado una señal favorable ( $E \mid H$ 
y $D \mid H$ ) o una señal desfavorable ( $E \mid L$ y $D \mid L$ ). Por ejemplo, los pagos esperados al efectuar una recomendación consistente con la señal son

$$
\Pi(E \mid H)=q_{1}(H) t_{1 H}+\left[1-q_{1}(H)\right] t_{1 L}
$$

$\mathrm{y}$

$$
\Pi(D \mid L)=\beta q_{2}(L) t_{2 H}+\beta\left[1-q_{2}(L)\right] t_{2 L}
$$

Definimos como costo delegación el pago esperado al experto:

$$
C D=p(H) \Pi(E \mid H)+p(L) \Pi(D \mid L)
$$

El contrato debe ser atractivo para el experto si éste decide esforzarse y efectuar la recomendación consistente con la señal que observa.

$$
C_{D}-e \geq 0
$$

El lado izquierdo es el el pago esperado si el experto decide participar, esforzarse y efectuar una recomendación consistente con la señal, menos el costo de esfuerzo: el lado derecho es la utilidad de reserva del agente, normalizada a cero.

El contrato también debe incentivar al experto a esforzarse por adquirir información. Por lo tanto, los pagos deben satisfacer la siguiente restricción de incentivos al esfuerzo:

$$
C_{D}-e \geq \max \{\Pi(E), \Pi(D)\}
$$

El lado derecho representa el máximo pago esperado que el experto puede obtener si debe efectuar una recomendación sin haberse informado. Si esta recomendación es ejecutar inmediatamente, el pago esperado es

$$
\Pi(E)=\lambda t_{1 H}+(1-\lambda) t_{1 L}
$$

y si la recomendación es diferir, el pago esperado es

$$
\Pi(D)=\beta \Lambda t_{2 H}+\beta(1-\Lambda) t_{2 L}
$$


La condición (IF) es equivalente a las dos condiciones siguientes:

$\mathrm{y}$

$$
C_{D}-e \geq \Pi(E)
$$

$$
C_{D}-e \geq \Pi(D)
$$

Además, el contrato debe incentivar al experto a efectuar una recomendación consistente con la señal observada. Por lo tanto, los pagos deben satisfacer las siguientes restricciones:

$$
\Pi(E \mid H) \geq \Pi(D \mid H)
$$

$\mathrm{y}$

$$
\Pi(D \mid L) \geq \Pi(E \mid L)
$$

La primera de estas dos restricciones establece que si el experto observa una señal favorable $(s=H)$, debe preferir débilmente recomendar la ejecución inmediata; similarmente, la segunda establece que si el experto observa una señal desfavorable, debe preferir débilmente recomendar la postergación de la decisión.

Finalmente, el contrato debe especificar pagos no negativos, debido a que el experto tiene responsabilidad limitada:

$$
t_{1 H}, t_{1 L}, t_{2 H}, t_{2 L} \geq 0
$$

Definición: El contrato es incentivo factible (FACT) si verifica las restricciones (PP), (IFE), (IFD), (RCE), (RCD) y (RL).

El problema del inversor es diseñar el contrato incentivo factible que maximize su utilidad esperada, que es

$$
p(H) \Pi_{I}(E \mid H)+p(L) \Pi_{I}(D \mid L)
$$

donde $\Pi_{I}(E \mid H)$ es el beneficio actual esperado, neto de pagos al experto, que obtiene el inversor al ejecutar inmediatamente el proyecto, si el experto luego de esforzarse y observar la señal favorable, así lo recomienda, y $\Pi_{I}(D \mid L)$ es 
el beneficio neto actual esperado de diferir la ejecución, si el experto luego de esforzarse y observar la señal desfavorable, recomienda esperar al siguiente periodo. Estos pagos son

$$
\Pi_{I}(E \mid H)=q_{1}(H)\left(G-t_{1 H}\right)+\left[1-q_{1}(H)\right]\left(-P-t_{1 L}\right)
$$

y

$$
\Pi_{I}(D \mid L)=\beta q_{2}(L)\left(G-t_{2 H}\right)-\beta t_{2 L}\left[1-q_{2}(L)\right]
$$

El estado de la naturaleza en $t=2$ es observable. Por lo tanto, el inversor óptimamente decide no ejecutar el proyecto si se observa la señal desfavorable, evitándose la pérdida $P$. No obstante, a pesar de que el proyecto no se ejecute, dejamos abierta la posibilidad de que el contrato incluya un pago al experto si en el segundo periodo el estado de la naturaleza es desfavorable.

Como el inversor es neutral al riesgo, podemos expresar los beneficios esperados de los pagos esperados al experto como $V$ - $C D$, donde

$$
V=p(H) q_{1}(H) G-p(H)\left[1-q_{1}(H)\right] P+\beta p(L) q_{2}(L) G
$$

es el valor de la opción real si el inversor fuera experto, es decir, tuviera la capacidad para adquirir información en el primer periodo. Como $V$ no depende de los pagos al experto, el problema del inversor puede expresarse como el de elegir los pagos al experto incentivo factibles que minimizan el costo de delegación:

$$
\min C_{D} \text { sujeto a }
$$

Las restricciones al esfuerzo implican las restricciones de consistencia en la recomendación. GM argumentan que si el experto prefiriera efectuar una recomendación inconsistente, no necesitaría la señal para hacerlo, por lo que no tendría sentido esforzarse para obtener dicha señal. Formalmente:

Lema 1. Si un contrato verifica las restricciones (IFE) y (IFD) entonces también verifica las restricciones (RCE) y (RCD).

Igualmente, las restricciones de esfuerzo implican la restricción de participación. 
Lema 2. Si un contrato verifica las restricciones (IFE) y (IFD) entonces también verifica la restricción (PP).

Como consecuencia, podemos omitir las restricciones de consistencia en la recomendación y la restricción de participación.

Definimos el costo de agencia como la diferencia entre el costo de delegación y el costo de esfuerzo del experto:

$$
C_{A}=C_{D}-e
$$

La siguiente proposición establece que el problema del inversor está bien definido, y caracteriza el contrato óptimo.

\section{Proposición 1.}

(i) Existe solución al problema de diseño óptimo del contrato.

(ii) Si $\vartheta_{2}(H)>\vartheta_{2}(L)$ y el experto recomienda la ejecución inmediata, el inversor sólo debe recompesar al experto si el proyecto tiene éxito, con el siguiente pago:

$$
t_{1 H}=\frac{e}{\lambda\left[\vartheta_{1}(H)-\vartheta_{2}(L)\right]}
$$

y si el experto recomienda la postergación de la decisión, el inversor sólo debe recompensar al experto si al resolverse la incertidumbre, el inversor observa que el estado de la naturaleza en $t=2$ es el desfavorable, dejando morir la opción. En tal caso, el pago debe ser

$$
t_{2 L}=\frac{e}{\beta(1-\Lambda)\left[\vartheta_{1}(H)-\vartheta_{2}(L)\right]}
$$

(iii) Si $\vartheta_{2}(H)<\vartheta_{2}(L)$, y el experto recomienda la ejecución inmediata, el inversor sólo debe recompesar al experto si el proyecto tiene éxito, con el siguiente pago:

$$
t_{1 H}=\frac{e}{\lambda\left[\vartheta_{1}(H)-\vartheta_{2}(H)\right]}
$$

y si el experto recomienda la postergación de la decisión, el inversor sólo debe recompensar al experto si al resolverse la incertidumbre, el inversor observa que el estado de la naturaleza en $t=2$ es el favorable, ejerciendo la opción. En tal caso, el pago debe ser

$$
t_{2 H}=\frac{e}{\beta \Lambda\left[\vartheta_{1}(H)-\vartheta_{2}(H)\right]}
$$


La demostración de la proposición puede verse en el apéndice. La misma se basa en construir un problema (llamado problema primal) cuyo dual es el problema del inversor, mostrar que la solución al primal es positiva, y vincularla con las holguras de las restricciones al esfuerzo utilizando la teoría de dualidad en programación lineal.

Las holguras de las restricciones de incentivos al esfuerzo son

$$
h_{E}^{*}=C_{D}-e-\Pi(E)
$$

$\mathrm{y}$

$$
h_{D}^{*}=C_{D}-e-\Pi(D)
$$

El teorema de la holgura complementaria (cfr. Vanderbei, 2007, TH 5.3) establece que el producto entre cada variable solución del primal y la correspondiente holgura del dual debe ser igual a cero. En el apéndice mostramos que la solución al primal es positiva. Como consecuencia, las holguras complementarias son cero, por lo que las restricciones de incentivos al esfuerzo se cumplen con igualdad. Por lo tanto, la diferencia entre el costo de delegación y el costo de esfuerzo es

$$
C_{D}-e=\Pi(E)=\Pi(D)
$$

que es positiva en el óptimo, indicando que sea cual fuere su recomendación, el pago esperado del experto debe compensarlo por el esfuerzo, e incentivarlo a efectuar una recomendación consistente con la señal observada con un monto adicional al costo de esfuerzo. Este monto adicional es el costo de agencia, y es igual a $\Pi(E)$ y а $\Pi(D)$ :

$$
C_{A}=\Pi(E)=\Pi(D)
$$

El inversor compensa al experto por su esfuerzo, y además ofrece la menor prima posible para asegurar consistencia en la recomendación. Esta consistencia en la recomendación se garantiza ofreciéndole al experto beneficios iguales a los que esperaría si efectúa una recomendación sin esforzarse. Además, los pagos óptimos son tales que si el experto no se esfuerza, estaría indiferente entre recomendar la ejecución inmediata o la postergación de la decisión. (En el óptimo, $\Pi(E)$ y $\Pi(D)$ son iguales; GM 
obtienen el mismo resultado en su setup de un periodo.) El siguiente corolario se deriva de la proposición anterior.

Corolario 1. El costo de agencia es positivo.

El contrato óptimo es consistente con un principio general en problemas de acción oculta: el pago debe estar vinculado a los eventos más informativos sobre el esfuerzo del agente. Concretamente, la actualización bayesiana que el inversor hace sobre la probabilidad de una recomendación de ejecución inmediata, consistente con la señal observada $(s=H)$, es mayor si el estado de la naturaleza es el favorable, debido a que la señal es informativa:

$$
\vartheta_{1}(H)=\theta>1 / 2>1-\theta=\vartheta_{1}(L)
$$

Siguiendo el mismo criterio, si el experto recomienda postergar la decisión, en el siguiente periodo se lo deberá recompensar en el evento más informativo sobre el esfuerzo del agente. Este evento puede ser tanto la ocurrencia del estado favorable como la ocurrencia del estado desfavorable (a diferencia del pago en el primer periodo en el que el evento más informativo es siempre el estado favorable). El inversor debe asociar el pago a la ocurrencia del estado favorable en el segundo periodo sólo si $\vartheta_{2}(H)<\vartheta_{2}(L)$, o equivalentemente, si $1-\vartheta_{2}(H)>1-\vartheta_{2}(L)$, es decir, sólo si el evento más informativo sobre la recepción de una señal desfavorable por parte del experto $(s=L)$ es la ocurrencia del estado favorable en el segundo periodo. Si $\vartheta_{2}(H)>\vartheta_{2}(L)$, el evento más informativo sobre la recepción de una señal desfavorable por parte del experto es la ocurrencia del estado desfavorable en el periodo $t=2$. En tal caso, el inversor debe recompensar al experto sólo si ocurre el estado desfavorable. Aunque puede parecer contraintuitivo, tiene sentido: la idea es incentivar al experto a recomendar la postergación del proyecto sólo si observa una señal desfavorable; si $\vartheta_{2}(H)>\vartheta_{2}(L)$, la probabilidad que el inversor asigna a la recepción de una señal favorable por parte del experto (y por lo tanto de haber hecho una recomendación inconsistente) es mayor luego de observar el estado favorable en el segundo periodo. Si quiere desincentivar esta recomendación inconsistente, el inversor no debe recompensar al experto en el estado favorable.

Notar que el pago al experto disminuye con aumentos en la diferencia $\vartheta_{1}(H)-\vartheta_{2}\left(S_{2}\right)$. Intuitivamente, si el experto recomienda la ejecución, 
el problema de asimetría informacional se agrava a menor contenido informativo de los resultados del proyecto sobre el esfuerzo del agente (a menor $\vartheta_{1}(H)$, por lo que el pago al experto debe aumentar; por otro lado, si el experto recomienda la postergación, el pago debe aumentar con $\vartheta_{2}\left(S_{2}\right)$, que es la probabilidad de que el experto haya observado una señal favorable, dado que el estado de la naturaleza en el segundo periodo es $S_{2}$. Mayores valores de esta probabilidad equivalen a menores valores de la probabilidad de que el experto haya observado una señal desfavorable en el primer periodo, y por lo tanto haya efectuado una recomendación consistente. En otras palabras, altos valores $\vartheta_{2}\left(S_{2}\right)$ significan que la ocurrencia del estado $S_{2}$ es poco informativo sobre la consistencia en la recomendación de esperar. Esto agrava el problema de asimetría informacional, por lo que el pago al experto debe aumentar.

\section{Conclusiones}

En el presente trabajo hemos obtenido el contrato óptimo que el dueño de una opción real debe proponer al experto para que involucre recursos costosos que le permitan recabar información de utilidad para decidir sobre el timing óptimo, y para que efectúe una recomendación consistente con la información recabada. Si el experto recomienda la ejecución inmediata, el contrato establece una recompensa sólo si el proyecto tiene éxito. Si en cambio el experto recomienda postergar la decisión en espera de mayor información, el pago en el segundo periodo debe hacerse contingente a la información que se revela al postergar el proyecto. El contrato puede considerar un pago si el proyecto se abandona, o si se ejecuta con éxito, dependiendo de cuál sea el estado más informativo sobre la acción del experto en el primer periodo. El pago esperado al experto es mayor que su costo de esfuerzo, ya que es necesaria una prima para incentivar al experto a revelar la información adquirida efectuando una recomendación consistente. 


\section{Referencias bibliográficas}

Dixit, A. K., R. S. Pindyck (1994), Investment under Uncertainty, Princeton University Press.

Gromb, D., D. Martimort (2007), “Collusion and the organization of delegated expertise", Journal of Economic Theory, vol. 137, núm. 1, pp. 271-299.

McDonald, R., D. Siegel (1986), “The value of waiting to invest", The Quarterly Journal of Economics, vol. 101, núm. 4, pp. 707-27.

Smith, H. T. J., L. Trigeorgis (2004), Strategic Investment: Real Options and Games, Princeton University Press.

Vanderbei, R. (2007), Linear Programming: Foundations and Extensions (International Series in Operations Research \& Management Science), 3rd edn. Springer.

\section{Apéndice}

A continuación se desarrollan las demostraciones de los resultados del trabajo. En lo que sigue, para simplificar la exposición de las funciones objetivo y de las restricciones, utilizamos la siguiente notación:

$$
\begin{aligned}
& a=\vartheta_{1}(H) \lambda=\theta \lambda \\
& b=\vartheta_{1}(L)(1-\lambda)=(1-\theta)(1-\lambda) \\
& c=\beta\left[1-\vartheta_{2}(H)\right] \Lambda=\beta\left[\lambda_{H}(1-\theta) \lambda+\left(1-\lambda_{L}\right) \theta(1-\lambda)\right] \\
& d=\beta\left[1-\vartheta_{2}(L)\right](1-\Lambda)=\beta\left[\left(1-\lambda_{H}\right)(1-\theta) \lambda+\lambda_{L} \theta(1-\lambda)\right] \\
& \left.f=\beta \vartheta_{2}(H)\right] \Lambda=\beta\left[\lambda_{H} \theta \lambda+\left(1-\lambda_{L}\right)(1-\theta)(1-\lambda)\right] \\
& \left.g=\beta \vartheta_{2}(L)\right](1-\Lambda)=\beta\left[\left(1-\lambda_{H}\right) \theta \lambda+\lambda_{L}(1-\theta)(1-\lambda)\right] \\
& j=\left[1-\vartheta_{1}(H)\right] \lambda=(1-\theta) \lambda \\
& k=\left[1-\vartheta_{1}(L)\right](1-\lambda)=\theta(1-\lambda)
\end{aligned}
$$


Lema 1. (IF implica IC y PP) El problema de diseño óptimo del contrato queda expresado de la siguiente manera:

$$
\begin{aligned}
& \min a t_{1 H}+b t_{1 L}+c t_{2 H}+d t_{2 L} \\
& a t_{1 H}+b t_{1 L}+c t_{2 H}+d t_{2 L}-e \geq 0 \\
& (a-\lambda) t_{1 H}+[b-(1-\lambda)] t_{1 L}+c t_{2 H}+d t_{2 L}-e \geq 0 \\
& a t_{1 H}+b t_{1 L}+(c-\beta \Lambda) t_{2 H}+[d-\beta(1-\Lambda)] t_{2 L}-e \geq 0 \\
& c t_{2 H}+d t_{2 L} \geq j t_{1 H}+k t_{1 L} \\
& a t_{1 H}+b t_{1 L} \geq f t_{2 H}+g t_{2 L} \\
& t_{1 H}, t_{1 L}, t_{2 H}, t_{2 L} \geq 0
\end{aligned}
$$

Como el costo de esfuerzo es no negativo, $c t_{2 H}+d t_{2 L} \geq c t_{2 H}+d t_{2 L}-e$. Suponer que (IFE) se verifica; entonces el lado derecho es mayor o igual a $j t_{1 H}+k t_{1 L}$; por lo tanto, también se verifica (RCD). Similarmente, si (IFD) se verifica, entonces

$$
a t_{1 H}+b t_{1 L} \geq a t_{1 H}+b t_{1 L}-e \geq(\beta \Lambda-c) t_{2 H}+[\beta(1-\Lambda)-d] t_{2 L}=f t_{2 H}+g t_{2 L}
$$

que implica que (RCE) también se verifica.

Lema 2. (IF implican PP) Si las restricciones al esfuerzo se cumplen, entonces

$$
a t_{1 H}+b t_{1 L}+c t_{2 H}+d t_{2 L}-e \geq \lambda t_{1 H}+(1-\lambda) t_{1 L}+e \geq e
$$

$\mathrm{y}$

$$
a t_{1 H}+b t_{1 L}+c t_{2 H}+d t_{2 L}-e \geq \beta \Lambda t_{2 H}+\left[d+\beta(1-\Lambda) t_{2 L}+e \geq e\right.
$$

donde las últimas desigualdades se deben a que los pagos son no negativos. Ambas implican (PP).

Proposición 1. (Existencia de solución - Contrato óptimo) Notar que el problema del inversor es el dual del siguiente problema primal: 
Esteban Colla

$\max e(x+y)$

sujeto a

$-(\lambda-a) x+a y \leq a$

$-[(1-\lambda)-b] x+b y \leq b$

$c x+(c-\beta \Lambda) y \leq c$

$[d-\beta(1-\Lambda)] x+d y \leq d$

$x, y \geq 0$

Este problema es factible (por ejemplo, $(0,0)$ verifica el conjunto de restricciones). Mostraremos que el problema primal está acotado, para lo cual expresamos la primera restricción como $y \leq 1+[(1-\theta) / \theta] x$ y la segunda como y $y \leq 1+[\theta /(1-\theta)] x$. Como la señal es informativa $\theta>1 / 2$, la primera condición implica la segunda que, por lo tanto, podemos omitir. Del mismo modo, la tercera y cuarta restricción pueden expresarse como $y \geq(c / f)(x-1)$ y $y \geq(d / g)(x-1)$. La recta $y=1+[\theta /(1-\theta)] x$ tiene ordenada al origen en $y=1$, y las rectas $y=(c / f)(x-1)$ y $y=(d / g)(x-1)$ tienen abcisa al origen en $x=1$. Notar que sólo estará activa la restricción correspondiente a la recta con la mayor de las pendientes. Por lo tanto, para probar que el problema está acotado, basta con demostrar que la mayor de las pendientes de estas rectas es mayor que $(1-\theta) / \theta$, es decir, basta con demostrar que $\max \{c / f, d / g\}>(1-\theta) / \theta$. Suponer que $\max \{c / f, d / g\}=c / f$. Entonces, si la señal es informativa,

$$
\begin{aligned}
& \theta>1 / 2 \Rightarrow \theta^{2}>(1-\theta)^{2} \\
& \Rightarrow \lambda_{H} \lambda \theta(1-\theta)+\left(1-\lambda_{L}\right)(1-\lambda) \theta^{2}>\lambda_{H} \lambda \theta(1-\theta)+\left(1-\lambda_{L}\right)(1-\lambda)(1-\theta)^{2} \\
& \Rightarrow c / f>(1-\theta) / \theta .
\end{aligned}
$$

Siguiendo el mismo procedimiento, $\max \{c / f, d / g\}=d / g$.

$$
\begin{aligned}
& \theta>1 / 2 \Rightarrow \theta^{2}>(1-\theta)^{2} \\
& \Rightarrow\left(1-\lambda_{H}\right) \lambda \theta(1-\theta)+\lambda_{L}(1-\lambda) \theta^{2}>\left(1-\lambda_{H}\right) \lambda \theta(1-\theta)+\lambda_{L}(1-\lambda)(1-\theta)^{2} \\
& \Rightarrow d / g>(1-\theta) / \theta .
\end{aligned}
$$


Por lo tanto, el problema está acotado. El teorema fundamental de la programación lineal (cfr. Vanderbei (2007) TH. 3.4) implica que el dual es factible; por lo tanto, existe solución al problema de diseño del contrato óptimo. La solución al problema primal satisface las siguientes restricciones con igualdad:

$$
\begin{gathered}
y=1+\xi x \\
y=\eta(x-1)
\end{gathered}
$$

donde $\xi=(1-\theta) / \theta$ y $\eta=\max \{c / f, d / g\}$. Por lo tanto,

$$
\begin{aligned}
& x^{*}=(1-\eta) /(\eta-\xi)>0 \\
& y^{*}=\eta(1-\xi) /(\eta-\xi)>0
\end{aligned}
$$

Las holguras del problema primal son

$$
\begin{aligned}
& \tau_{1 H} \equiv 1+\xi x^{*}-y^{*}=0 \\
& \tau_{1 L} \equiv 1+\frac{1}{\xi} x^{*}-y^{*}=\frac{\left(1-\xi^{2}\right)(1+\eta)}{\xi(\eta-\xi)}>0 \\
& \tau_{2 H} \equiv 1-x^{*}+\frac{f}{c} y^{*}=\frac{(1+\xi)\left(\frac{f}{c} \eta-1\right)}{\eta-\xi}>0 \\
& \tau_{2 L} \equiv 1-x^{*}+\frac{g}{d} y^{*}=\frac{(1+\xi)\left(\frac{g}{d} \eta-1\right)}{\eta-\xi}>0
\end{aligned}
$$

Tanto el problema primal como el dual son factibles. Notar que $\tau_{1 L}>0$ implica que $t_{1 L}=0$. Además, como $x^{*}$ y $y^{*}$ son mayores a cero, el teorema de la holgura complementaria (Vanderbei (2007), TH5.3) implica quelas restricciones de incentivos al esfuerzo se cumplen con igualdad. Si $\eta=c / f>d / g$, entonces $\tau_{2 L}>0$, y si $\eta=d / g>c / f$, entonces $\tau_{2 H}>0$. (Note que la desigualdad $c / f>(<) d / g$ es equivalente a $\vartheta_{2}(H)>(<) \vartheta_{2}(L)$. Por lo tanto, las restricciones 
de incentivos al esfuerzo conforman un sistema de dos ecuaciones con dos incógnitas, $t_{1 H}$ y $t_{2 H}$ o $t_{2 H}$ dependiendo de la comparación de $\vartheta_{2}(H)$ versus $\vartheta_{2}(L)$, cuya solución corresponde a los pagos de la proposición.

Corolario 1. (El costo de agencia es positivo) El corolario se deriva del teorema fuerte de dualidad (Vanderbei, 2007; TH 5.2), que establece que el costo de delegación del inversor, función objetivo del problema de minimización evaluada en el óptimo, es igual a la función objetivo del primal evaluada en la solución. Sustituyendo $x^{*}$ y $y^{*}$ en la función objetivo del problema primal obtenemos el costo de delegación $\left.C_{D}=[(1+2 \eta-\xi \eta) / \eta-\xi)\right]$ e. Notar que $\eta(\xi-1)-\xi$ es menor a 0 . Por lo tanto, $1>\eta(\xi-1)-\xi$ que implica $1+2 \eta-\eta \xi>\eta-\xi$ o $(1+2 \eta-\eta \xi) /(\eta-\xi)>1$. El costo de agencia es

$$
C_{A}=e\{[(1+2 \eta-\xi \eta) /(\eta-\xi)]-1\}=e(1+\eta+\xi-\xi \eta) /(\eta-\xi)
$$

que es positivo. 